\title{
Melt-layer ejection and material changes of three different tungsten materials under high heat-flux conditions in the tokamak edge plasma of TEXTOR
}

\author{
J.W. Coenen ${ }^{1}$, V. Philipps ${ }^{1}$, S. Brezinsek ${ }^{1}$, G. Pintsuk ${ }^{1}$, I. Uytdenhouwen ${ }^{2}$,

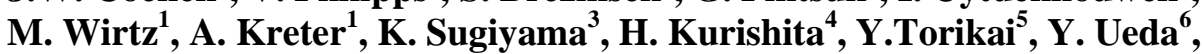 \\ U. Samm ${ }^{1}$ and the TEXTOR-Team ${ }^{1}$ \\ ${ }^{1}$ Institute for Energy and Climate Research, Forschungszentrum Jülich, EURATOM \\ Association,Trilateral Euregio Cluster, Jülich, Germany \\ ${ }^{2}$ SCK-CEN, Belgian Nuclear Research Centre, Association EURATOM, Partner In the \\ Trilateral Euregio Cluster, Mol, Belgium \\ ${ }^{3}$ Max-Planck-Institut für Plasmaphysik, EURATOM Association, Garching, Germany \\ ${ }^{4}$ Hydrogen Isotope Research Center, University of Toyama, Japan \\ ${ }^{5}$ International Research Center for Nuclear Materials Science, Institute for Materials \\ Research (IMR), Tohoku University, Oarai, Ibaraki 311-1313, Japan \\ ${ }^{6}$ Graduate School of Engineering, Osaka University, Osaka 565-0871, Japan \\ E-mail: j.w.coenen@fz-juelich.de
}

\begin{abstract}
.
The behaviour of tungsten (W) plasma-facing components (PFCs) has been investigated in the plasma edge of the TEXTOR tokamak to study melt-layer ejection, macroscopic tungsten erosion from the melt layer as well as the changes of material properties such as grain-size and abundance of voids or bubbles. The parallel heat flux at the radial position of the exposed tungsten tile in the plasma ranges around $q_{\|} \sim 45 \mathrm{MW} \mathrm{m}^{-2}$ causing samples to be exposed at an impact angle of $35^{\circ}$ to $20-30 \mathrm{MW} \mathrm{m}^{-2}$. Locally the temperature reached up to $6000 \mathrm{~K}$, high levels of evaporation and boiling are causing significant erosion in the form of continuous fine spray or droplet ejection. The amount offine-spray tungsten emission depends strongly on the material properties: in the case of the tungsten-tantalum alloy the effect of spraying and droplet emission is significantly higher at even low temperatures when compared with regular tungsten or even ultra-high purity tungsten which shows almost no spraying at all. Differences in the material composition, grain structure and size may be related to the different evolution of macroscopic erosion. In addition the re-solidified material is studied and strong differences in terms of re-crystallized grain size and evolution of the grain structure and grain orientation are observed. The build up of large voids has been observed.
\end{abstract}

\section{Introduction}

ThePlasma-wall interaction (PWI) is one of the main aspects to be considered towards ITER and a future fusion reactor. Due to low physical sputtering and favourable thermo-mechanical properties high- $\mathrm{Z}$ materials are the potential wall candidates for reactor-like machines. $\mathrm{W}$ has the highest melting point $(3695 \mathrm{~K})$ of all elements and behaves rather benign after neutron irradiation. W was chosen as part of the ITER divertor design and is the material used for the activated phase. The use of 
tungsten, however, is leading to additional constraints. Melting under uncontrolled conditions and the associated material re-distribution and material loss places limits on power handling, lifetime and plasma operation. Steady-state loads at the W surface of the ITER divertor are estimated to values of $10 \mathrm{MW} \mathrm{m} \mathrm{m}^{-2}$ at the strike point area under normal operation conditions in the non-activated phase. Transients, such as ELMs, can reach $\sim \mathrm{GW} \mathrm{m^{-2 }}$ on a short timescale (ms). Any loss of positioning control, uncontrolled transients as well as possible misalignment of target mono-blocks can thus potentially cause melting [1,2] subsequently deforming the PFCs leading up to peak power-loads well above the set limits. Occurrence of melt layers, melt-layer motion, re-crystallization, void formation and material ejection can drastically decrease the PFC lifetime, lead to strong plasma contamination and degradation of the power-handling capabilities. This paper presents experiments on the melt behaviour of different tungsten grades under tokamak conditions focussing on the influence of material choices with respect to material losses.

The paper is structured as follows, after the short introduction into the topic a section describing the macroscopic melt-layer losses by means of spectroscopic measurements and 2D-images is given followed by a section considering material properties as one possible reason for the differences in tungsten sources followed by a summary and conclusions.

\section{Experiments and setup}

Experiments were carried out by introducing a limiter made of a graphite carrier with dedicated tungsten plates within inlets (compare [3-5]) into the deuterium plasma by means of the PWI-test facilities [6] at TEXTOR $(R=1.75 \mathrm{~m} a=0.47 \mathrm{~cm})$ [7]. Two materials are being tested apart from the previously used materials (Plansee or A.L.M.T. co [5]): ultra-high purity tungsten (WUHP 99.9999\% tungsten) and a tungsten-tantalum alloy (WTA5\%) with $5 \mathrm{wt} \%$ tantalum included.

The $\mathrm{W}$ plates were mounted with minimal thermal contact to facilitate melting. The plasma parameters are $I_{\mathrm{p}}=350 \mathrm{kA}, B_{\mathrm{T}}=2.25 \mathrm{~T}, n_{\mathrm{e}}=3.5 \times 10^{19} \mathrm{~m}^{-3}, P_{\mathrm{NBI}}=1.2 \mathrm{MW}$. The limiters are introduced from the bottom of the machine through a lock system. The limiters were moved stepwise $\left(\Delta_{r} \sim 0.2 \mathrm{~mm}\right)$, between pulses, towards the LCFS and finally beyond, until melting occurred at $46.2 \mathrm{~cm} .\left(T_{\mathrm{e}}(a) \sim 70 \mathrm{eV}, n_{\mathrm{e}}(a) \sim 1 \times 10^{19} \mathrm{~m}^{-3}, q_{\mathrm{l}} \sim 60 \mathrm{MW} \mathrm{m}{ }^{-2}\right.$, He-beam data [8]). Similar experiments have been discussed in [3-5] where regular tungsten grades were exposed in contrast to the special materials used here.

An extensive set of diagnostics is used. Temperature measurements are performed via a single colour pyrometer $(1$ spot, $\lambda=3.25 \mu \mathrm{m})$ (figure 1$)$ and a near infrared camera $(\lambda=(1.0-1.7) \mu \mathrm{m})$. W emissivity dependences are taken into account with respect to wavelength and temperature $[9,10]$. The uncertainty in terms of temperature measurement is $\sim 300 \mathrm{~K}$ taking into account also an uncertainty in the emissivity and surface structure.

Spectroscopic data are available from the $\mathrm{W}$ continuum $(\sim 5 \mathrm{~nm}(\mathrm{VUV}))$ radiating at the core of TEXTOR $\left(T_{\mathrm{e}}=1 \mathrm{keV}\right.$ [11]) as well as from the local emission of the $400.8 \mathrm{~nm} \mathrm{~W}$ I line. Details of the spectroscopic systems can be found in [12,13]. Interference filters of $1.5 \mathrm{~nm}$ bandwidth have been used. 2D video cameras (20 ms time resolution) from the side (figure 1) are used to observe the $\mathrm{W}_{\mathrm{I}}$ influx and the particle ejection. The average temperature rises up to $3500 \mathrm{~K}$ with an average heat flux

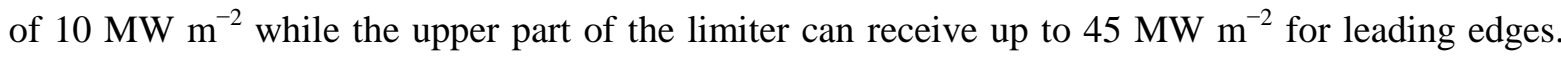
The typical heat flux is $\sim 20 \mathrm{MW} \mathrm{m}^{-2}$ for a duration of $5 \mathrm{~s}$, peak temperatures from IR measurements reach $4000-6000 \mathrm{~K}$. Strong cooling by evaporation and radiation leads to an equilibrium temperature level and an effective decrease in the heat flux to the limiter in the melting phase with constant 
plasma conditions. This includes probably some level of interaction of the emitted $\mathrm{W}$ with the plasma leading up to a decrease in core plasma temperature [5].

\section{Material spraying and splashing}

During experiments with regular Plansee or A.L.M.T. co grade tungsten a strong plasma contamination was found as described in $[3,5]$ which can, during steady-state melting, emit a large amount of material (W) due to evaporation and in addition by macroscopic loss of droplets during socalled spraying and splashing events. This is either a continuous emission of small droplets $(\sim 4 \mu \mathrm{m})$ in the case of spraying or larger droplets in the case of splashing. The red curve (\# 112062) in figure 2 represents the $\mathrm{W}_{\mathrm{I}}(400.8 \mathrm{~nm})$ line emission during experiments with strong spraying and additional splash events (bumps) demonstrating the strong W source during these events. In comparison in grey (\# 114597) and black (\#114598) the WI emission for experiments using ultra-high purity tungsten (WUHP) is shown under identical heat-flux conditions. A tungsten source is still visible; however, no strong increase with time and power is observed. The source remains an order of magnitude lower or less. The maximum temperature in both cases is roughly 4500-5000 K. All curves are derived from the $400.8 \mathrm{~nm}$ tungsten emission utilizing a photo-efficiency (S/XB) of 100. The $\mathrm{W}$ source consists of a mixture of evaporation and macroscopic losses. The possible mechanism for macroscopic meltlayer losses is described in [14-16] accounting for the possibility of bubble boiling of the material with the bubbles typically forming on impurity seeds or grain boundaries. This implies that high purity materials with large grains should suffer less from spraying events than impurity containing small grained materials. In addition to the spectroscopic signal shown in figure 2, figure 3 displays a composite of the complete melt exposure of WUHP. Apart from the typically overexposed areas close to the target visible due to Planck radiation no spraying behaviour can be observed which is consistent with the spectroscopic observations. In contrast to this, the tungsten-tantalum alloy shows a drastically different behaviour. Figure 4 shows the strong macroscopic melt-layer loss terminating subsequently the discharge. From left to right the shot overview is given followed by images of two distinct phases, a tungsten fine spraying phase and the strong particle ejection. In figure 5 , time traces of the spatially integrated $400.8 \mathrm{~nm} \mathrm{~W}$-emission are given. The green and black curves represent melt exposures at the same radial position $(r=46.6 \mathrm{~cm})$ while the other exposures are at $(\# 114158) r=$ $46.8 \mathrm{~cm}$ or $r=48 \mathrm{~cm}$.

Pulse \#114155 was disrupted because of strong tungsten contamination due to splashing while the other discharges show a more continuos tungsten source in the form of fine-spray tungsten emission. The typical melt exposure is performed at $r=46.6 \mathrm{~cm}$ whereas the non-tungsten reference is achieved by retracting the limiter to $r=48 \mathrm{~cm}$. With decreasing minor radius, the heat-flux increases towards the LCFS at $r=46.6 \mathrm{~cm}$. This clearly shows that while spraying is influencing the plasma, splashing can severely impact the discharge duration and stability [5]. Figure 6 compares the time traces of the W-emission observed from melt exposures of WUHP as well as WTa5\%. For the WUHP a maximum of up to $5000 \mathrm{~K}$ in surface temperature is measured while for the WTa5\% a significant tungsten source is already observed with a maximum close to the melting point $\left(T_{\text {melt }}=\right.$ $3695 \mathrm{~K})$.

As has been stated before from modelling [14,15], evaporation and macroscopic losses play different roles when comparing different materials. This can be clearly seen when comparing figures 7 and 6. For the WUHP case, the W source is roughly 50\% lower than for the WTa5\% case while the 
temperatures are quite different $(T \sim 1000 \mathrm{~K})$ during the exposure. This large difference can mean a strong influence on the evaporation of tungsten during the pulse.

This indicates that when assuming (from observation) no spraying in the WUHP case and pure evaporation behaviour, that the spraying is dominantly contributing to the signal observed during shot \#114159, especially during the two bumps visible. Figure 7 presents the necessary information. While during the whole shot spraying is present, two phases of intensive particle ejection are observed which correspond in time with the measured spatially integrated W flux (figure 5). Droplet emission is potentially connected to, on the one hand, a melt-layer instability [16] in the case of larger droplets and in the case of spraying to so-called bubble boiling of the material $[14,15]$. It is assumed that larger droplets evolve from a shear instability, e.g. Kelvin-Helmholtz, leading up to filaments and droplets leaving the surface, while smaller droplets are produced by local boiling of the material beneath the surfaces and expulsion of small particles during the collapse of surface bubbles (figure 8).

The main plasma contamination has been observed by means of the VUV tungsten quasicontinuum emission at $\sim 5 \mathrm{~nm}$, which is a measure for the tungsten in the TEXTOR core plasma ( $1 \mathrm{keV}$ ). Figure 9 shows two time traces, the one for WUHP (black) and WTa5\% (red). The data show strong intensity peaks during the evolution of the discharge (figure 6) following the increase in the local source during the WTa5\% melt exposure, while for the ultra-high purity tungsten only background levels are seen.

\section{Material structure evolution}

In order to understand the underlying mechanism the $\mathrm{W}$ material structure is studied before and after the melt exposure. Obviously, the material purity is an important parameter determining the material ejection by spraying and splashing, since a purified sample such as WUHP is not showing spraying or splashing, while the tantalum containing more impure material does even show stronger emissions when compared with similar melt exposures of regular tungsten grades [5].

In [15], bubble boiling is seen as the most likely explanation for macroscopic melt-layer ejection in the form of spraying (figure 8) apart from the so-called Kelvin-Helmholtz-instability when considering fast moving melt layers not present under TEXTOR conditions [16,17]. Bubble formation requires a seed to form, such as grain boundaries, impurity clusters or microscopic irregularities. Figures $10(a)$ and $(b)$ can give some hints for the reasons of different material ejection behaviour. For the tungsten-tantalum alloy one starts out with a fine grained material which even after being re-crystallized or re-solidified remains much more fine-structured as the WUHP, even adding additional irregular features and layers. Figures $10(a)$ and $(b)$ show the evolution from a virgin material sample via re-crystallized structures towards the partly re-crystallized, partly resolidified material from the TEXTOR exposures.

The ultra-high purity tungsten starts out with grains already much larger than the ones in the WTa5 material. This is probably due to the fact that grain growth is heavily influenced by the impurities in the material. The tungsten-tantalum alloy shows a rather small grain structure and in addition suffers from much larger re-crystallization than seen in the WUHP. In addition, the number of voids is much more pronounced in the WTa5 material than in the WUHP (cf figures $10(b)$ and 14) an indication for a potentially facilitated boiling due to impurity content and material structure. These structural differences together with the work performed in [15] must account for the difference in the tungsten source behaviour. 


\subsection{Cracking and material strength}

As described in the previous section WUHP is more benign in terms of spraying and splashing phenomena. However, material ejection is only one aspect and material cracking and stability behaviour are the others. Visible inspection (figures $11(a)$ and $(b)$ ) after exposure shows that WUHP and WTa5\% have a totally different cracking behaviour. While WUHP is resilient to the thermal stresses induced during melting and cool-down, WTa5\% does crack all through the sample under quiescent steady-state conditions. This is connected to the different strength of the two materials [1820]. This difference is visible from the steady-state exposures in TEXTOR, additional transient heat flux tests reveal cracking for all re-crystallized materials.

High heat-load tests on the material have been performed using the electron beam facility JUDITH, following tests on the ITER reference materials [18,21]. Several spots on one of the exposed and damaged WUHP samples have been used to compare the response of undamaged (figure 12) with damaged (molten) material (figure 13).

In the case of the JUDITH test facility, electrons are generated by a tungsten cathode and accelerated. Although the acceleration voltage is high, the beam penetration was limited to about 5-7 $\mu \mathrm{m}$ in the solid $\mathrm{W}$ according to a numerical simulation. The beam parameters are $P_{\text {beam }}=60 \mathrm{~kW}, V_{\text {acc. }}$

$=120 \mathrm{kV}, D_{\text {beam }} \sim 1 \mathrm{~mm}, P_{\text {dens }}<1.13 \mathrm{GW} \mathrm{m}{ }^{-2}, t_{\text {exposure }}>1 \mathrm{~ms}, n_{\text {exp. }}=100, A_{\text {scan }}=16 \mathrm{~mm}^{2}$. Measurements are taken to compare with studies already performed on regular tungsten grades [22]. Figure 12 shows the unmolten area exposed at $200^{\circ} \mathrm{C}$ to $1.13 \mathrm{GW} \mathrm{m}^{-2}$, representing a typical unmitigated ELM load, while figure 13 shows the re-solidified, re-crystallized area under the same conditions. In the case of the unmolten area, only surface modifications such as roughening are observed, visible in the slight discolouration, while for the re-solidified part strong cracking is observed up to the point that the crack boundaries are lifted up to $60 \mu \mathrm{m}$. This demonstrates that the re-solidified material can behave much worse under additional transient loads than the previously benign original material. Melting of PFC will not only increase the material erosion by melt-layer ejection and material sublimation, but can also lead to enhanced material loss due to the degradation of the heat response capability of the re-solidified material. This is particularly visible in figure 14 . The sample already presented in figures 13 and 12 is analysed further. A cut is shown through the parts exposed to $1.13 \mathrm{GW} \mathrm{m}^{-2}$ (at $200^{\circ} \mathrm{C}$ ) at two locations. Spot 1 positioned on the molten and resolidified material and spot 2 located on the re-crystallized but not molten material. In the whole area as seen before (figure $10(b)$ ) no voids or similar structure is apparent apart from the exposed spots. A tendency of decreasing tolerance of transients with increasing level of re-crystallization or even melting is observed. While the reference spot (figure 12) showed only surface modification, spots 1 and 2 show strong deep cracking and loose grains and in the case of spot 2 even the tendency of material loss on a large scale.

\section{Conclusion}

Experiments on the melt behaviour of different tungsten grades and $\mathrm{W}-\mathrm{Ta}$ alloys have been performed in the TEXTOR tokamak under quasi-steady-state melt conditions. Under these conditions, fine-spray $\mathrm{W}$ melt ejection has been identified as a new melt ejection mechanism. The result of this work shows that the $\mathrm{W}$ spraying depends strongly on the material properties, with no or very little spraying for ultra-high purity $\mathrm{W}$, a medium spray for conventional $\mathrm{W}$ materials $[3,5]$ and strong spraying for the $\mathrm{W}-\mathrm{Ta}$ alloys used in this work. Modelling work $[14,15]$ shows that bubble boiling is 
one of the favourable explanations for $\mathrm{W}$ spray behaviour observed here. Taking the mechanism of bubble coalescence and formation into account it seems reasonable that large grained highly purified materials allow for less bubble formation in the molten material than a small grained impurity enriched material. The comparison between especially ultra-high purity tungsten and the tungstentantalum alloy samples clearly points into this direction. Apart from the observation of the $\mathrm{W}$ melt spray-emission, large droplets are observed occasionally in the TEXTOR experiments. The occurrence of these large droplets is irregular but very critical for the stability of the TEXTOR plasma. Large droplets often lead to a sudden $\mathrm{W}$ accumulation and subsequent discharge termination. Machine operation is not hampered by the occurrence of fine spray apart from a decrease in core temperature, and multiple consecutive melt exposures can be performed. The occurrence of these droplets is not in line with the existing understanding of linear Kelvin-Helmholtz instabilities [17] pointing rather to a more complex mechanism as presented by non-linear modelling [16]. Thus, the mechanics of melt-layer ejection are not yet fully understood. After the TEXTOR melt exposures high heat-flux tests with electron beam impact have been performed on the unmolten and resolidified ultra-high purity $\mathrm{W}$ material sample to simulate transient power-fluxes under reactor relevant conditions. The data show a reduced damage threshold of the re-solidified material. In addition to the further understanding of the material loss mechanism the work presented here also points towards a problem for material development for future devices. Even highly adapted materials can during and after melting cause severe impact on the machine performance by either having a low tolerance with respect to material ejection or due to the fact that the re-solidified material does not even come close to the pre-designed material properties chosen.

\section{Acknowledgements}

This work, supported by the European Communities under the contract of Association between EURATOM/FZJ, was carried out within the framework of the EFDA Task Force on plasma-wall interactions. The views and opinions expressed herein do not necessarily reflect those of the European Commission. Special thanks to the team of the IEK-2 for their effort in supporting experiments and post-mortem analysis. 


\section{References}

[1] Pitts R.A. et al 2011 Physics basis and design of ITER plasma-facing components J. Nucl. Mater. 415 957-64

[2] Neu R.L. 2010 Experience with high-Z plasma-facing materials and extrapolation to future devices IEEE Trans. Plasma Sci. 38 453-60

[3] Coenen J.W. et al and the Textor-Team 2011 Tungsten melt layer motion and splashing on castellated tungsten surfaces at the tokamak TEXTOR J. Nucl. Mater. 415 78-82

[4] Coenen J.W., Philipps V., Brezinsek S., Bazylev B., Kreter A. Hirai T., Laengner M., Tanabe T., Ueda Y., Samm U. and the TEXTOR Research Team 2010 Analysis of tungsten melt layer motion and splashing under tokamak conditions at textor Proc. 23rd Int. Conf. on Fusion Energy 2010 (Daejeon, South Korea, 2010) (Vienna: IAEA) CDROM file EXD/ 6-1 and http://wwwnaweb.iaea.org/napc/physics/FEC/FEC2010/html/index.htm

[5] Coenen J.W., Philipps V., Brezinsek S., Bazylev B., Kreter A., Hirai T., Laengner M., Tanabe T., Ueda Y., Samm U. and the TEXTOR Research Team 2011 Analysis of tungsten melt layer motion and splashing under tokamak conditions at TEXTOR Nucl. Fusion $\mathbf{5 1} 083008$

[6] Schweer B. et al 2005 Limiter lock systems at TEXTOR: flexible tools for plasma-wall investigation Fusion Sci. Technol. 47 138-45

[7] Neubauer O., Czymek G., Giesen B., Huttemann P.W., Sauer M., Schalt W. and Schruff J. 2005 Design features of the tokamak TEXTOR Fusion Sci. Technol. 47 76-86

[8] Schmitz O. et al 2008 Status of electron temperature and density measurement with beam emission spectroscopy on thermal helium at TEXTOR Plasma Phys. Control. Fusion 50115004

[9] Sergienko G. 2010 Tungsten emissivity, private communications, Forschungszentrum Juelich GmbH

[10] Lide D.R. and Frederikse H.P.R. (ed) 1994-95 CRC Handbook of Chemistry and Physics (Boca Raton, FL: CRC Press)

[11] Puetterich T., Neu R., Dux R., Whiteford A.D., O’Mullane M.G. and the ASDEX Upgrade Team 2008 Modelling of measured tungsten spectra from ASDEX Upgrade and predictions for ITER Plasma Phys. Control. Fusion 50085016

[12] Brezinsek S., Sergienko G., Pospieszczyk A., Mertens P., Samm U. and Greenland P.T. 2005 Characterization of the deuterium recycling flux in front of a graphite surface in the TEXTOR tokamak Plasma Phys. Control. Fusion 47 615-34

[13] Pospieszczyk A. 2005 Spectroscopic diagnostics of tokamak edge plasmas Phys. Scr. T119 71-82

[14] Shi Y., Miloshevsky G. and Hassanein A. 2011 Theoretical studies of macroscopic erosion mechanisms of melt layers developed on plasma facing components J. Nucl. Mater. 412 123-8

[15] Shi Y., Miloshevsky G. and Hassanein A. 2011 Boiling induced macroscopic erosion of plasma facing components in fusion devices Fusion Eng. Des. 86 155-62

[16] Miloshevsky G. and Hassanein A. 2010 Modelling of Kelvin-Helmholtz instability and splashing of melt layers from plasma-facing components in tokamaks under plasma impact Nucl, Fusion $\mathbf{5 0}$ 115005

[17] Bazylev B., Janeschitz G., Landman I., Loarte A., Klimov N.S., Podkovyrovd V.L. and Safronov V.M. 2009 Experimental and theoretical investigation of droplet emission from tungsten melt layer Fusion Eng. Des. 84 441-5

[18] Linke J. et al 2011 Performance of different tungsten grades under transient thermal loads Nucl, Fusion 51073017 
[19] Wirtz M., Linke J., Pintsuk G. and Uytdenhouwen I. 2011 Comparison of the thermal shock performance of different tungsten grades and the influence of microstructure on the damage behaviour Workshop on Plasma Facing Materials and Components (Rosenheim, Germany 2011) (Poster)

[20] Wirtz M., Linke J., Pintsuk G. and Uytdenhouwen I. 2011 Comparison of the thermal shock performance of different tungsten grades and the influence of microstructure on the damage behaviour Phys. Scr. submitted

[21] Hirai T., Pintsuk G., Linke J. and Batilliot M. 2009 Cracking failure study of ITER-reference tungsten grade under single pulse thermal shock loads at elevated temperatures J. Nucl. Mater. 390$391751-4$

[22] Coenen J.W., Philipps V., Brezinsek S., Pintsuk G., Tanabe T., Ueda Y., Samm U. and the TEXTOR Team 2011 Analysis of structural changes and high-heat-flux tests on pre-damaged tungsten from tokamak melt experiments Phys. Scr. at press 


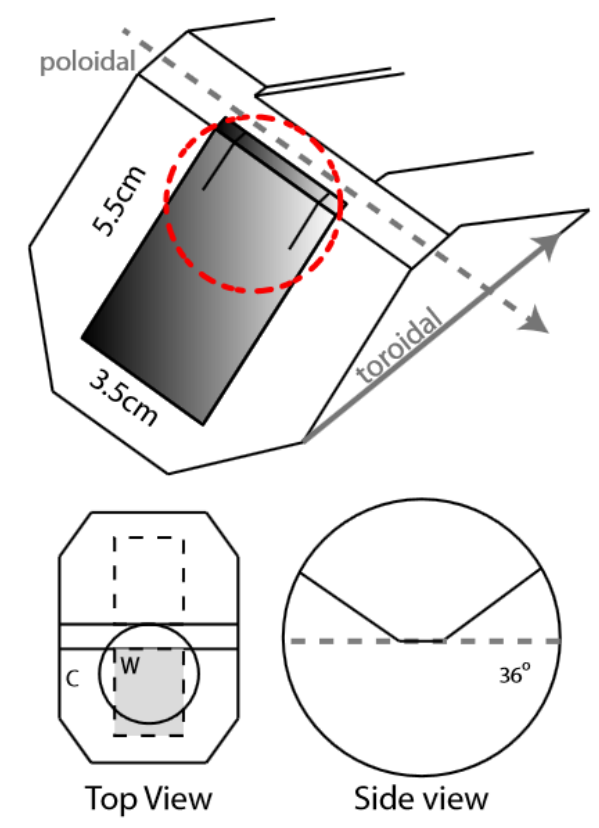

Figure 1.

Experimental setup for one tungsten sample installed on one side of the limiter. The red circle denotes pyrometer view.

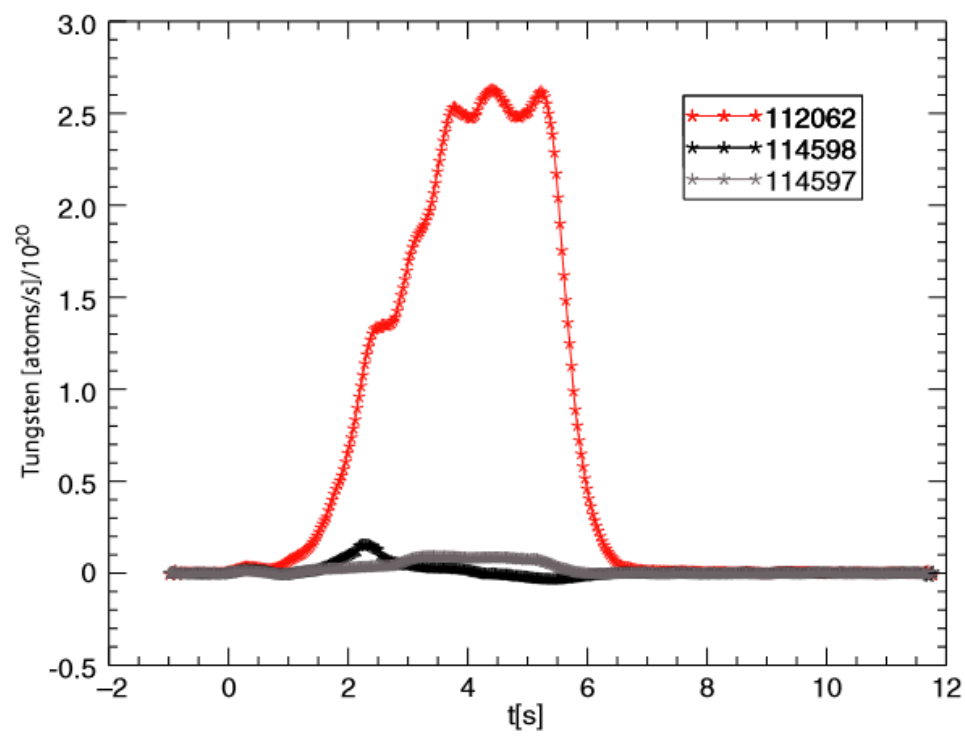

Figure 2.

Local tungsten source for two different materials: industry-grade W (red) and WUHP (black, grey). 


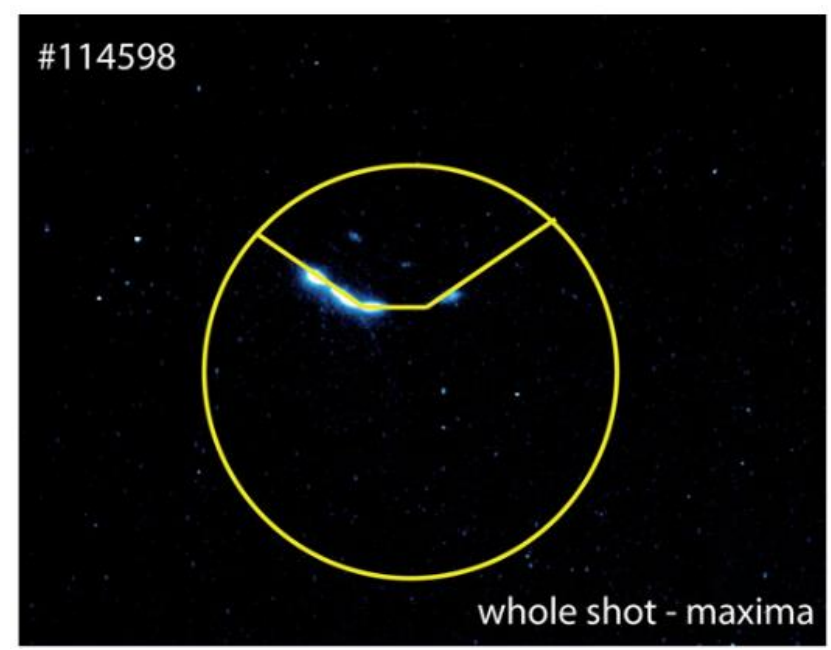

Figure 3.

Composite of W-emission during WUHP melting (\# 114598)
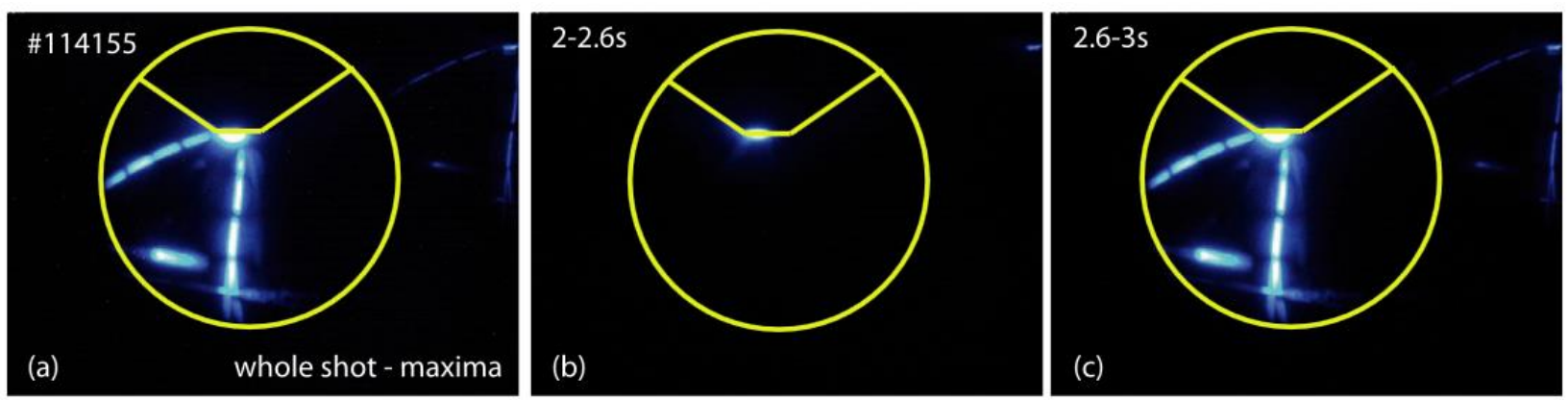

\section{Figure 4.}

W-emission during initial melt exposures on the tungsten-tantalum alloy (\#114155). Figure (a) represents the overall composite of all maxima observed, $(b)$ shows the fine spraying (cloudy-fine traces) phase, $(c)$ the splashing and ejection phase (big trace). 


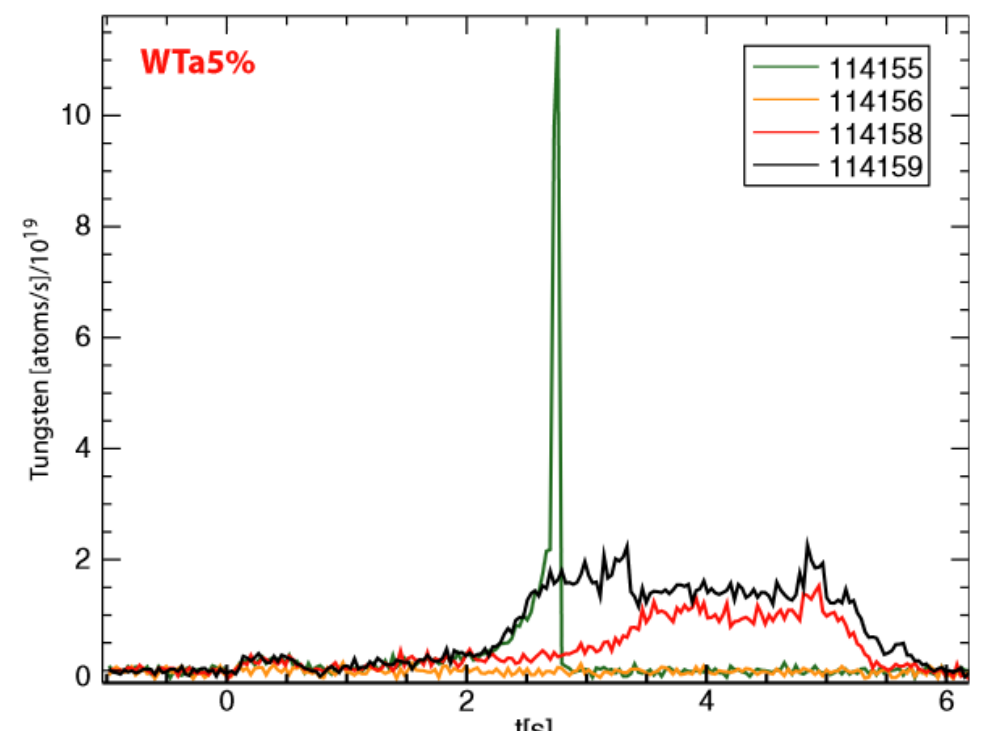

$\mathrm{t}[\mathrm{s}]$

\section{Figure 5.}

Local tungsten source during melt exposure of WTa5 at different radial positions (heat-flux). Black /green $-r=46.6$ (maximum heat-flux)

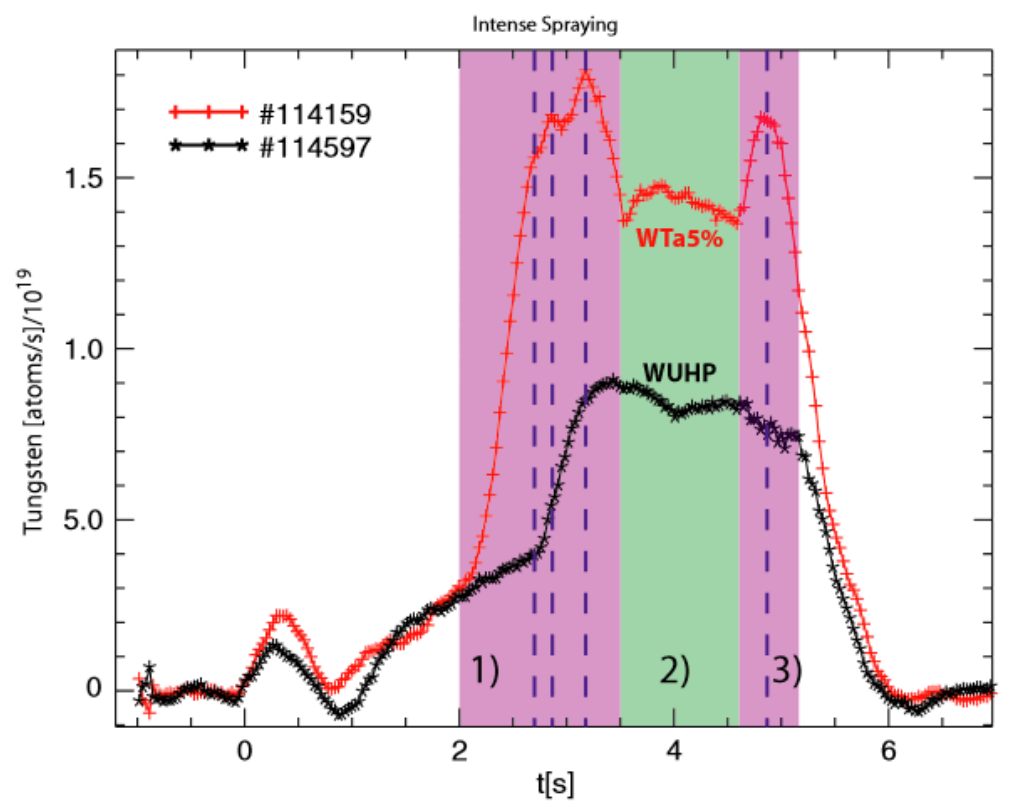

Figure 6.

Local tungsten source during comparable WTa5 and WUHp exposures are $r=46.6 \mathrm{~cm}$. Blue lines indicate ejection events. Phases 1-3 correspond to the images in figure 7 

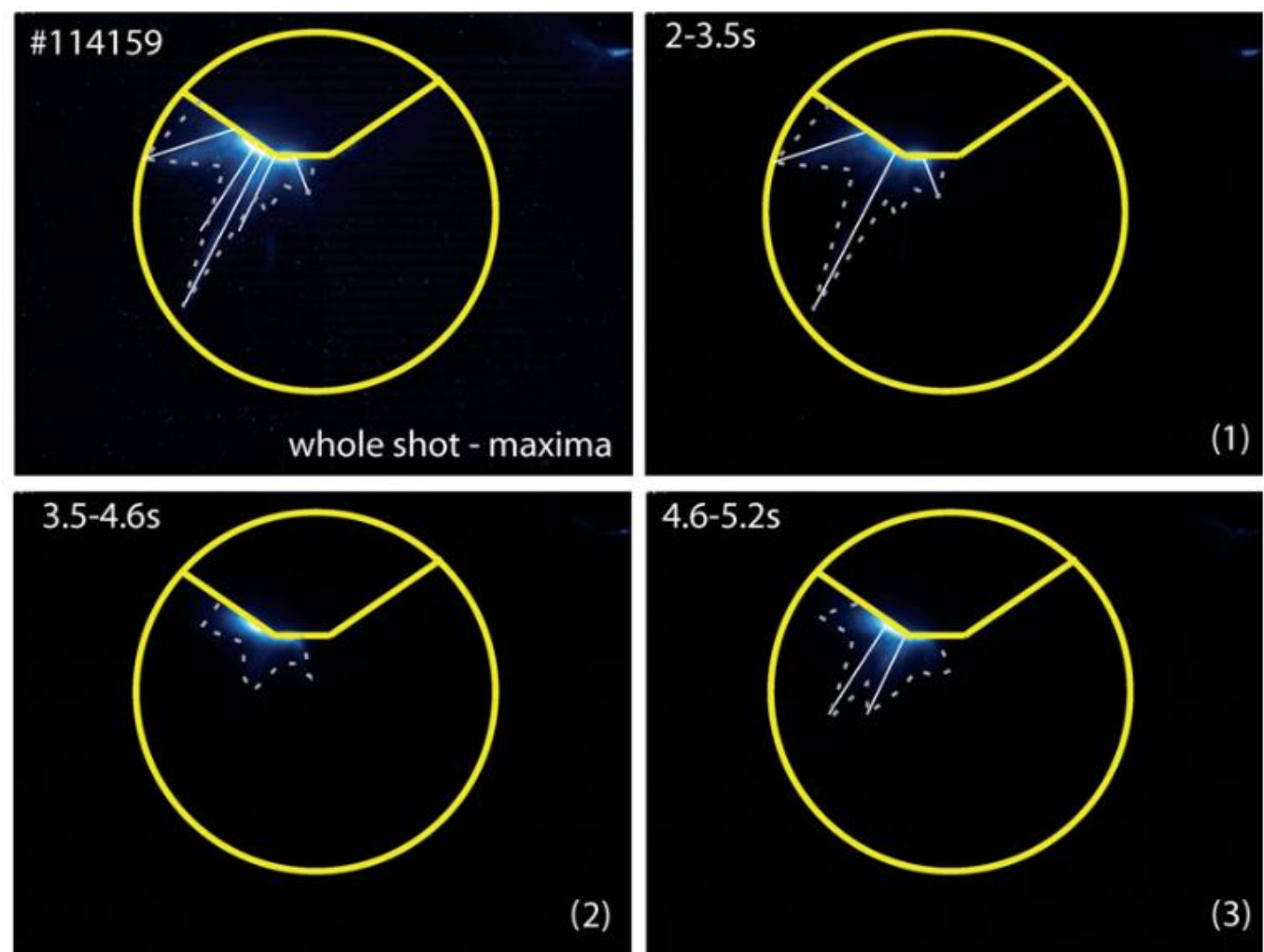

\section{Figure 7.}

W-emission during further melt exposures on the tungsten-tantalum alloy (\#114159). Images (1)-(3) are composites of the timeframes given (cf figure 6). Fine spray is enclosed and larger ejections are highlighted by grey lines.

(1)

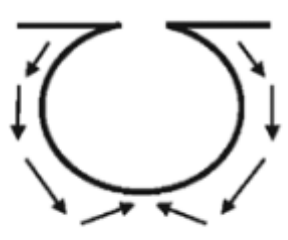

(2)

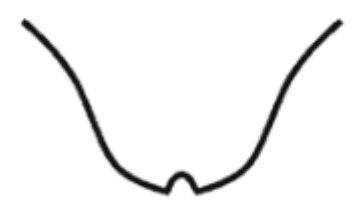

(3)

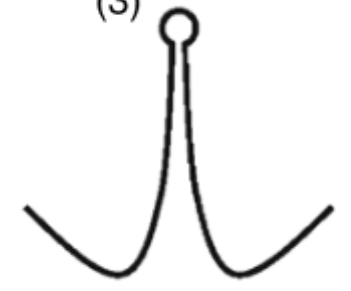

(4)

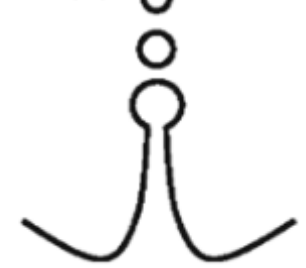

Figure 8.

Droplet emission from bubble boiling as described in [15]. Evolution from left (1) to right (4). 


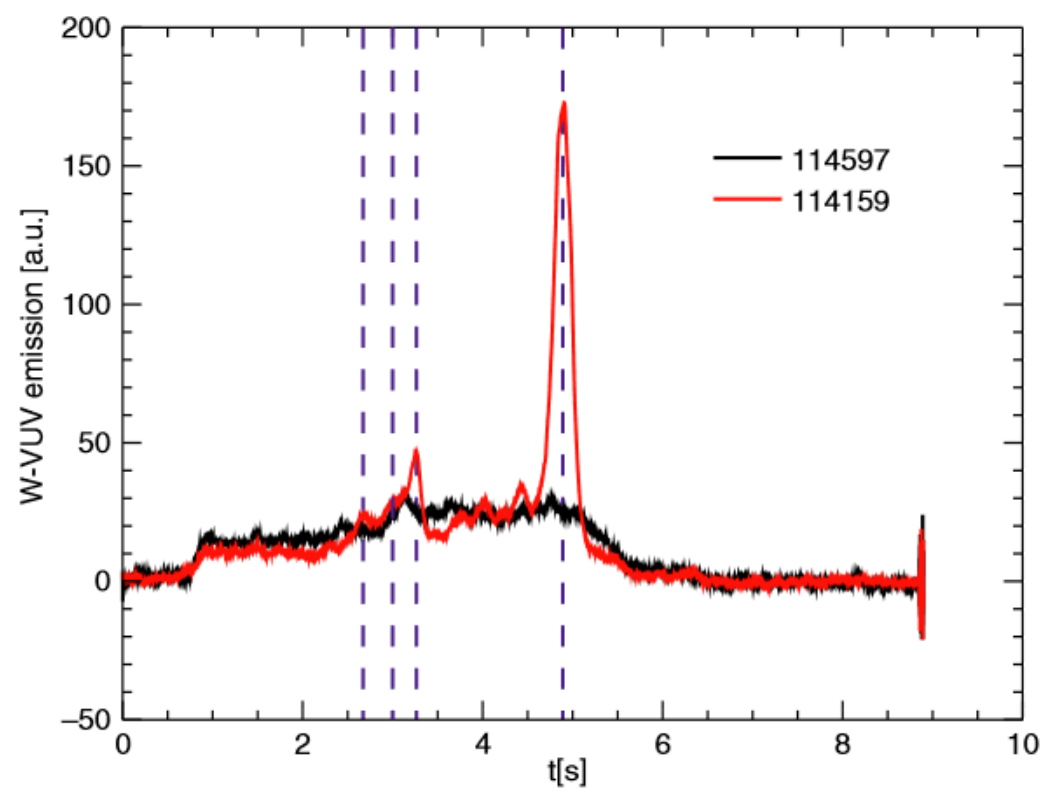

Figure 9.

Tungsten VUV emission during melt exposures of WUHP (\#114597) and WTa5\% (\#114159). Blue lines indicate material ejections. 

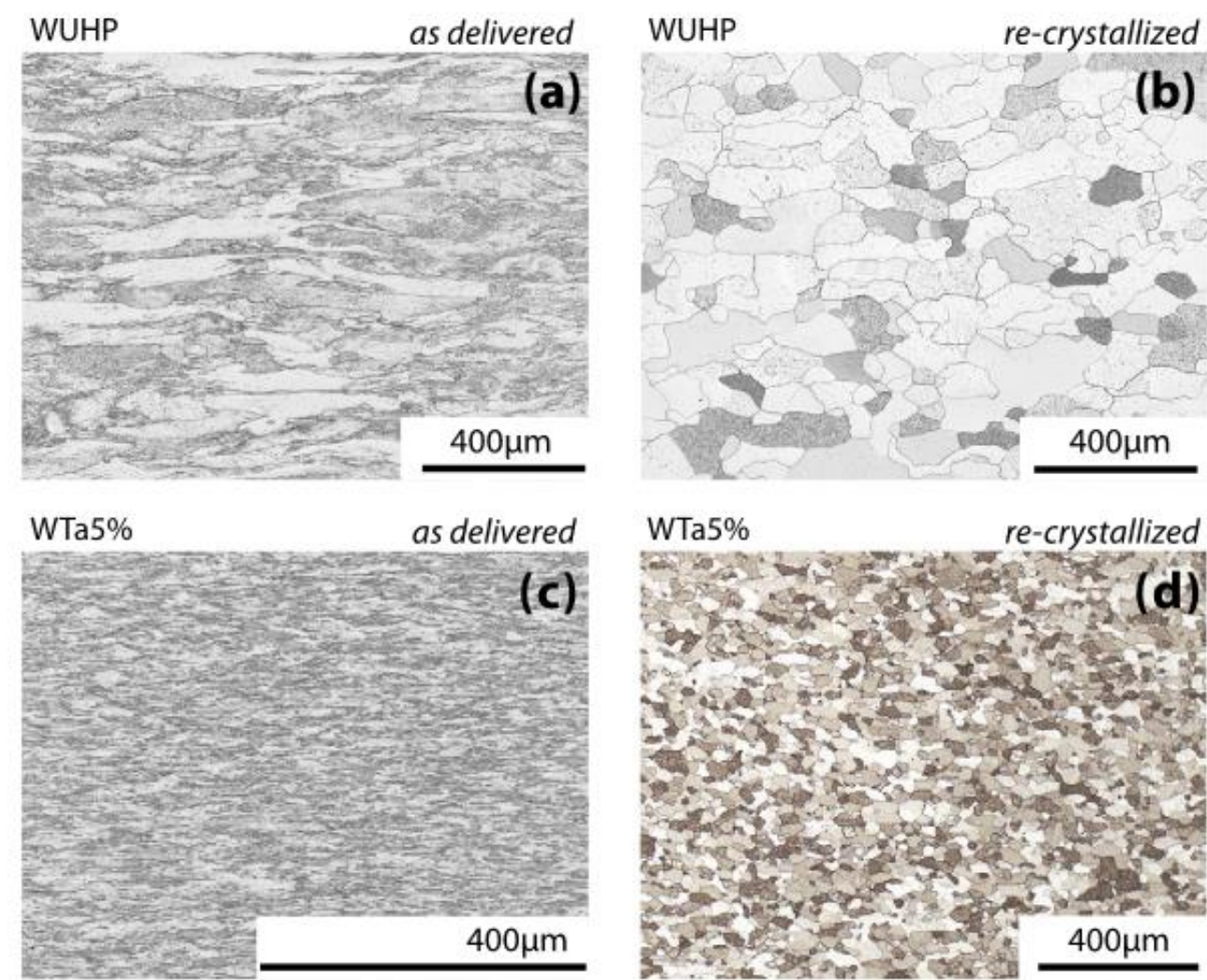

(a)

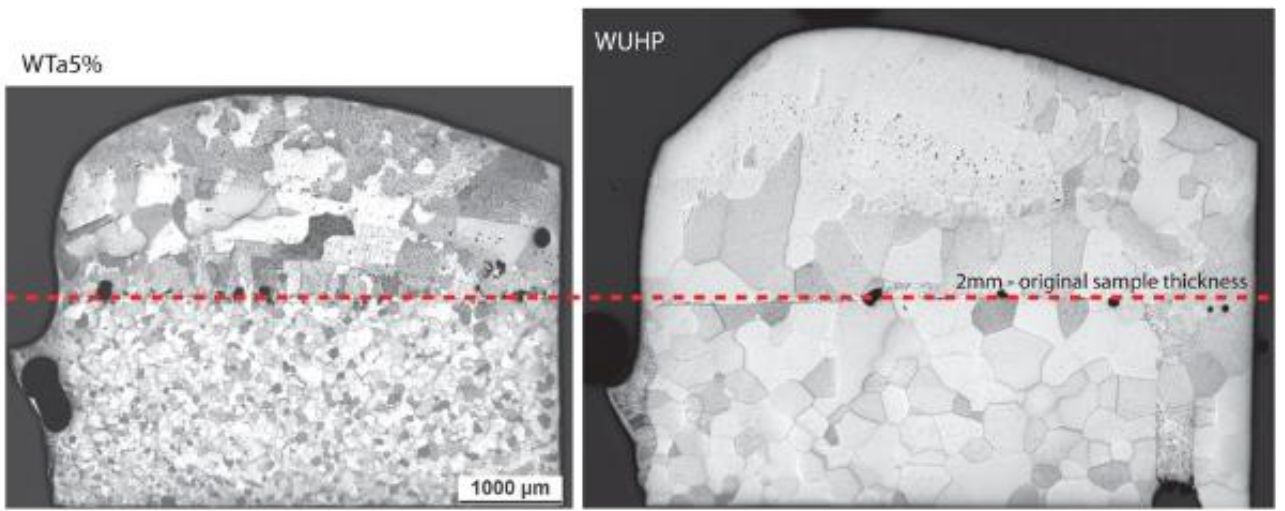

(b)

Figure 10.

Material structure of WUHP and WTa5\% before, 'during' and after exposure. 


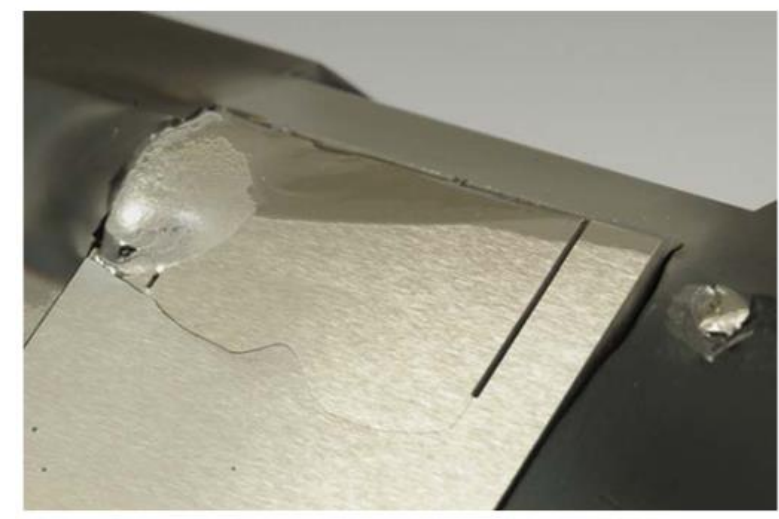

(a)

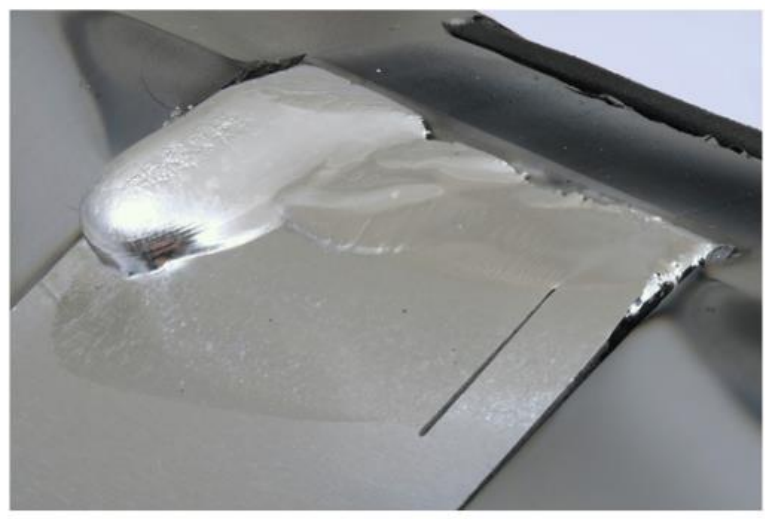

(b)

\section{Figure 11.}

Material re-distribution and cracking after steady-state melt exposures.

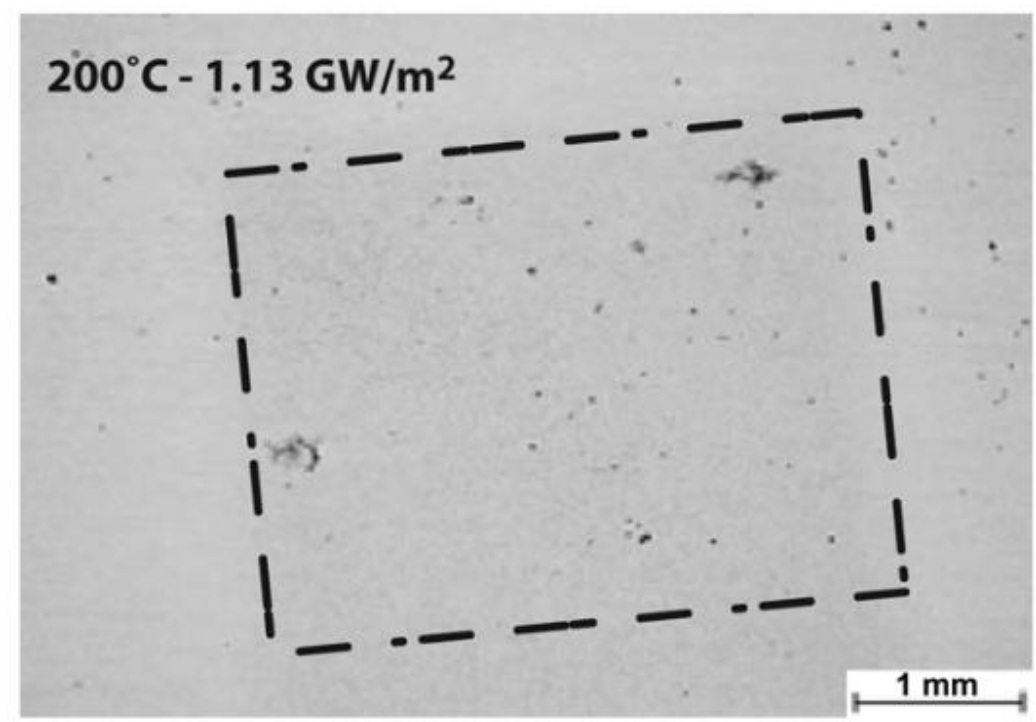

\section{Figure 12.}

Transient heat-flux test on the non-molten reference part of the WUHP sample. The dashed square indicates the footprint of the electron beam (reference spot). 


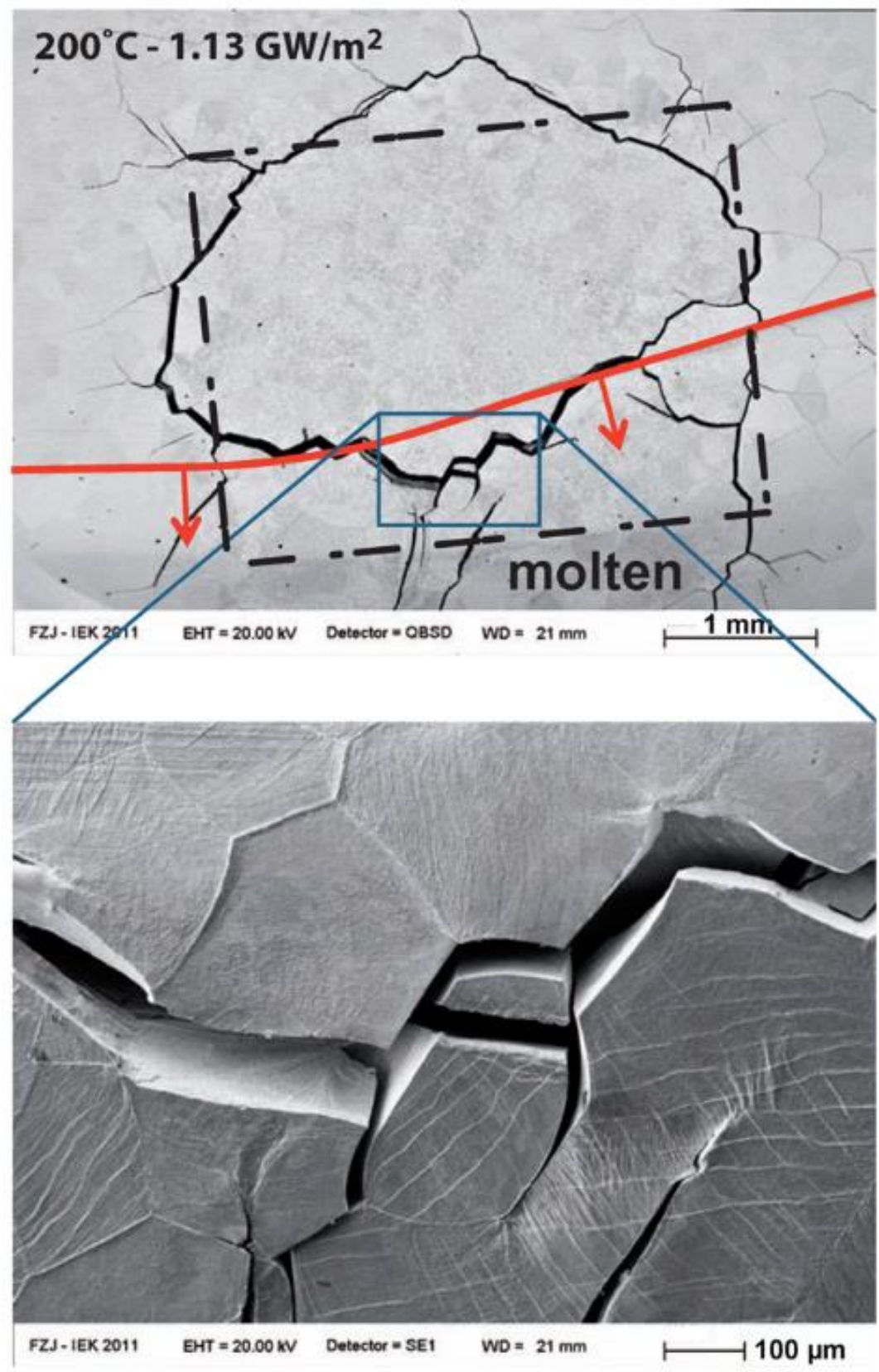

Figure 13.

Transient heat-flux test on molten, re-crystallized WUHP. The dashed square indicates the footprint of the electron beam (spot 1). The red line represents the molten to unmolten transition line. 